\title{
Smart monitoring system of Najran dam
}

\author{
A. H. M. Almawgani, M. A. Alshorman, M. A. Alyami, H. M. Alhmammi,
}

D. S. Almahri, M. A. Alkhuraym, A. H. Alwasel

Electrical Engineering Department, College of Engineering, Najran University, Saudi Arabia

\section{Article Info}

Article history:

Received Mar 20, 2019

Revised Jan 19, 2020

Accepted Mar 30, 2020

Keywords:

Arduino UNO

Dam gate

Flood

Monitoring system

Sensors

\begin{abstract}
Najran city faces the flood situation every year due to intensive rain and climatic disturbances. Flooding also causes loss of money, along with loss of life and property and the destruction of agriculture and livestock. Thus, this project proposes a conceptual framework with three main phases: monitoring the water level inside the dam and level in water stream before and after the dam, controlling the opening and closing of the dam gate and measuring the water pressure at the dam barrier. In the case of high water level dam is monitored through water sensors placed at the top of the dam and then these sensors give a reference to the stepper motor and the flow of the stepper motor is controlled by the dam gate. The experimental results shows that the proposed system has the capability to reliably tackle the flood water. It can accurately measure the water level and control the gate of dam as soon as the level of the water reaches to danger level and water pressure at the barrier is measured by the sensor. The developed real-time monitoring system in Najran dam will help authorities to take preventive actions to deal with flood disaster.
\end{abstract}

Copyright () 2020 Institute of Advanced Engineering and Science. All rights reserved.

\section{Corresponding Author:}

A. H. M. Almawgani,

Department of Electrical Engineering, College of Engineering,

Najran University,

Najran, Saudi Arabia.

Email: ahalmawgani@nu.edu.sa

\section{INTRODUCTION}

Najran Valley is one of the largest and most important valleys in the Arabian Peninsula and its tributaries come from the Hijaz Mountains and hills surrounding the area and extends a distance of 180 kilometers to the east, starting from its mouth in the plains and it ends in the sands of the Empty Quarter. The average displayed in the plains 1000 meters also bisects the valley city of Najran in two parts and is considered the source value of the goods and development of the region of Najran. In addition to being the main source of water supply for the region, it brings large amounts of silt deposited in the plains and supply water to the farm located on its banks in aid fertility. The agriculture in the valley of Najran on what is available in the underground water of the ebb and flow depending on the rainy seasons. Often exposed in the valley of the drought because of the lack of rain, the opposite has happened that is weeping floods destroy farms and property without the ability to restrain the water or control and take advantage of them. From this came the importance of building the dam, which is one of the largest dams in the Kingdom of Saudi Arabia, which is located just 15 kilometers from the Najran city. The Najran Valley Dam is an arch dam on vale Najran around $15 \mathrm{~km}$ southwest of Najran in Najran Province of southwest Saudi Arabia [1]. It has several purpose to include water supply, flood control and groundwater recharge. Its collects run-off and sediment in the vale and helps release it downstream slowly throughout the year.

Floods have devastating consequences and can have effects on the economy, environment and people. During floods (especially flash floods), roads, bridges, farms, houses and automobiles are destroyed [2]. The environment also suffers when floods occur. To prevent these effects, barrages and water dams are constructed to store the excess amount of water in the catchment area and proper water 
management is required for storing and releasing of water at barrages during floods. The water management cannot be achieved by using manual mechanism during floods and it required automatic detection and controlling. However, water management could be performed by automatic opening the barrage gates according to flow rate of water [3]. This system could prevent any danger that might happen to the dam based on measuring the water pressure on the dam, water level and forecasting on the status of the dam and prevent any danger that might happen to the dam. In the existing monitoring system of the Najran Dam, the water level is being monitored through human observation and gates are operated manually. The current system may cause delays in keeping pace with the situation of the dam and many disasters.

The problem in the current system may cause delays in keeping pace with the situation of the dam and many disasters can occur. To solve this issue, an automatic method has been developed in this research which has the capability to monitor the water level without human involvement. Effect of flooding in Najran area in which they occur, causing an imbalance in the ecological balance by affecting the ecosystem components and their effects are attributable to the mount of quantity and speed of flow. Flooding has many negative effects on people's lives, such as house demolitions and sabotage of roads and there is loss of large number of human and animal life.

When forecasting the flood baptizing to unload part of the dam reservoirs and lakes of water to reduce the water pressure with the start of the flood. So, as to exploit the updated storage capacity at the growing volume of flood water through the waterway to control the excess water and directed toward dam reservoirs and lakes to maintain the disposal capacity of the stream and reduce water leakage outside its borders. It can also exploit the valleys, lakes and reservoirs in the arid basin near the sinkhole areas to drain excess water and reduce the maximum discharge pressure for the Hungarian President. Embedded system is the combination of hardware and software co-design.

Embedded systems has a vital role in engineering design process for efficient analysis and effective ration. From data analysis to hardware work. Due to time complexity in electronic aspects embedded systems have become a major part of our daily life. Our work describes the design of an embedded system for the "embedded Dam control system" [4]. The water level and rate of inflow is detected based on the feedback from the sensors used. Based on this data, the level of water at dam gate can be automatically controlled using a DC motor. When the Dam gate is opened i.e. if the water level of the Dam exceeds then automatically Dam gate will be open [5]. That control the gates of the dam limit the damage in the course of the floods and not sabotage. Also This system measuring the water pressure on the dam. Where he does not have a dam in Najran any talk that measures the water level of a dam entered.

In [6], introduced the notion of water level monitoring and management within the context of electrical conductivity of the water. The authors motivated by the technological affordances of mobile devices and they believe that water level management approach would help in reducing the home power consumption and as well as water overflow; investigated the microcontroller based water level sensing and controlling in a wired and wireless environment. The research result was a flexible, economical and easy configurable system designed on a low cost PIC16F84A microcontroller and finally, proposed a web and cellular based monitoring service protocol to determine and sense water level globally.

Some of the new existing methods for measuring the river flow are the moving boat method, ultrasonic method and electromagnetic method. The water currents and levels are measured at far away from the barrage by flow sensor and level is measured at barrage by the level sensor [3]. In [7] proposed a system for effective control of Hydropower Dam Spillway using PLC/SCADA system. Recently manufacturing company has installed a PLC based control system for controlling the operation of dam gates [7]. In [3], proposed system improve safety conditions and optimum water usage. The level which exists at barrage is sensed by using the multipoint level sensor. So that there will be a reduction of damage caused due to floods. In [8], proposed a PC based dam control model wherein they introduced the concept of division of reservoirs into upper and lower reservoirs and efficiently use the dam water. The overall control was through PC and needed an operator. It consist of microcontroller and a PC for the mode that microcontroller don't support. So this reservoir sub division model can be merged with our system to get efficient resource conserving results. However, none of these studies have sends SMS to phones which present on the banks of the streams before or after the dam to warning them of the danger of rising water levels on their lives.

The advantages of the proposed system is that it works to monitor the dam and open the dam gate automatically instead of controlling manually. It also monitors the level of water flow to and control the flow of water when opening gates. In the case of high water level in the streams of water flow the proposed system sends SMS to phones which present on the banks of the streams before or after the dam. The purpose of sending SMS to phones passing or static in the course of the flow of water to warning them of the danger of rising water levels on their lives. This feature is not available in any of the references pervious references. 
Moreover, the proposed monitoring dam system combined with flood early warning system before and after the dam, which is advantage compared to other research works.

\section{METHODOLOGY}

\subsection{Flood early warning system of najran dam}

With the flood early warning system of Najran dam system, immediate action can be carried out in order to save lives and minimize the damage caused by flood disaster. With warning SMS that is broadcasted simultaneously based on user mobility and send SMS to monitoring system of Najran dam. The process of this system depends on the water level either if it becomes dangerous or in a safe condition [9-11]. The flood early warning system used Arduino Uno R3, GSM shield, LCD, Water level sensor and Water flow sensor as shown in Figure 1. Arduino is an open-source platform [12] used for constructing and programing electronics circuits [13-26]. The Arduino is utilized to analyze the data that will be received from the sensors that should be planted before and after the dam area and send it to the monitoring dam system to take the right decision such as open or close the dam gate and send SMS to people to give them a chance for preparing to move to a safe place as shown in Figure 2. It is guarantee that the information is delivered to the communities and passersby that reside within the affected area and monitoring system to open or close the dam gate, which at the same time helps local authorities to accelerate the process of evacuating flood victims to the relief center. Furthermore, with the presence of an effective early flood warning system, the process of monitoring level and flow water become automated, allowing proper management plan to be carried out before, during and after the occurrence of flood disaster.

Flood early Warning system of Najran dam depends on flow and level of flood. When the gates of the dam open, the system measures the speed, quantity and level of the water, so that if it reaches the danger stage, the alarm whistle is activated and sends a message via the mobile. Also the system instruct before dam to measure water stream flow and level of flood and send the data to control unit in monitoring system. The flow chart of the flood early warning system of Najran dam has been shown in Figure 2 and Circuit design has been shown in Figure 2. In case of implementation of the project on the real world will be determined the number and numbers of telephones located on the water stream before and after the dam in case of rising water level in one of the water stream through cooperation with telecommunications companies operating.

\subsection{Developed monitoring dam system}

The monitoring dam system develop mechatronics based system, which detect the water level inside dam, water stream level before and after dam and thereby control the movement of gates automatically in real-time which offers flexibility. This system consist of a set of sensors like water level sensors to Arduino by electrical wires, as well as connecting to the pressure sensitive Arduino by wire electrical connection and wireless inputs from flood early warning system before and after dam that send water stream flow level and intensity as discussed in Section 2.1. The output from Arduino is connected to LCD to display the water level as well as pressure and conducts Arduino to DC motor through ULN driver to control the opening and closing of the dam gate. The ULN driver that in turn control the operation of the stepper motor i.e. switches on the stepper motor moving it in a clockwise or counterclockwise direction. The system connection shown in Figure 3 and Figure 4.

Figure 5 below shows the circuit diagram for the developed system. Project work system based on a number of steps to reach the desired goals of the system:

- Receive signal from flood early warning system include the level and intensity of the flow of water stream before and after the dam.

- Sensors are planting the water level at a depth of dam.

- Sensitive sensor when the water signal sent to Arduino.

- The Arduino processes the signal and decision-making based on Figure 6, Table 1 and Table 2.

- After the signal processing are presents the water level on the display (LCD).

- The arrival of water to the third level is Arduino send a signal to the DC motor, where the engine in the controlled gate (open or closed).

- Cultivation pressure sensor in the middle of the dam barrier and is sensitive to take the signal and send it to the Arduino and then the Arduino address signal and displayed on the display (LCD).

The Najran dam monitoring system works based on water sensors are placed on three equal dimensions of the height of the dam prototype as shown in Figure 7 and level of water in water stream before and after dam. The decisions of gate control will be taken by the system depend on the water levels as shown in Table 1. Table 2 and Figure 6. Figure 8 shows the experimental setup of circuit connection of flood early warning system. 


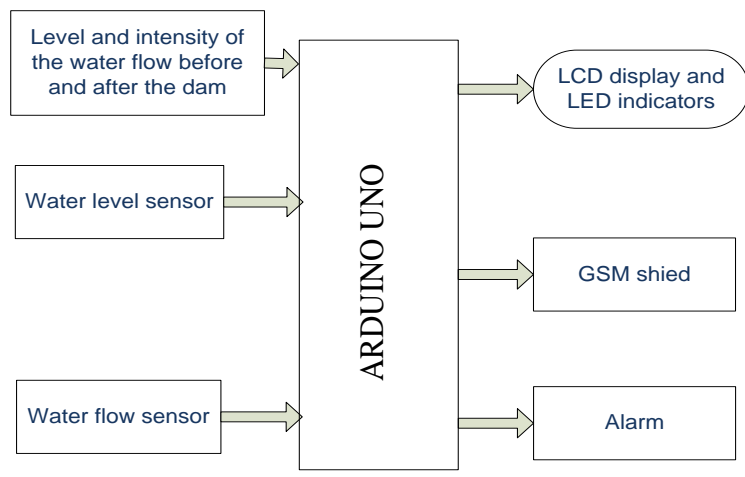

Figure 1. Block diagram of flood early warning system of Najran dam

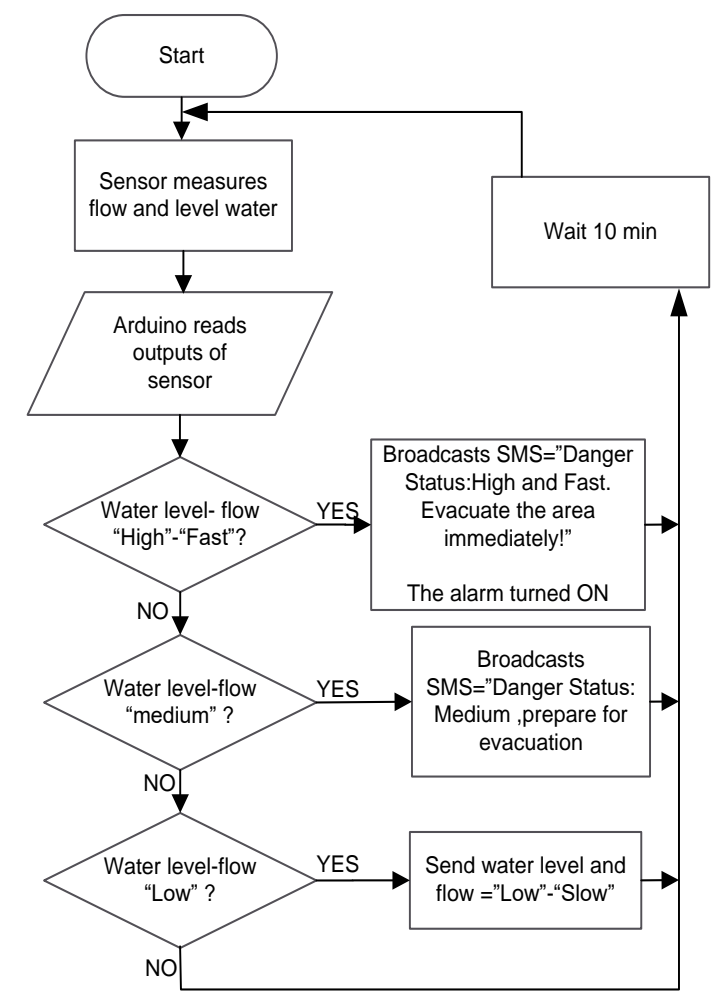

Figure 2. Flowchart of flood early warning system of Najran dam system

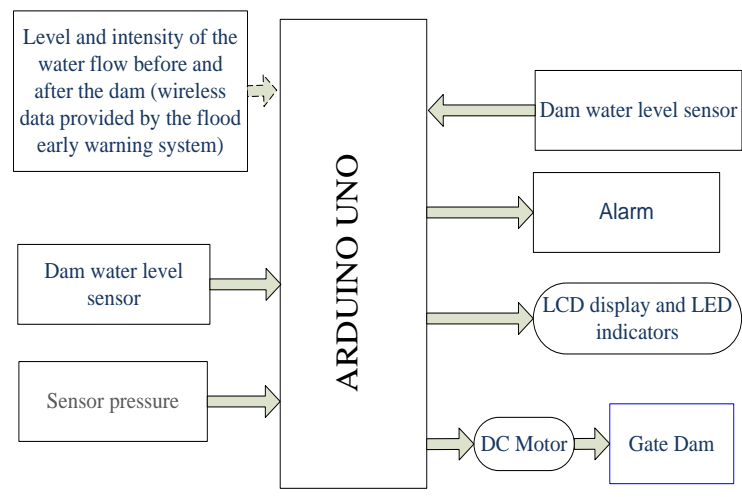

Figure 3. Block diagram of developed monitoring system of Najran dam 

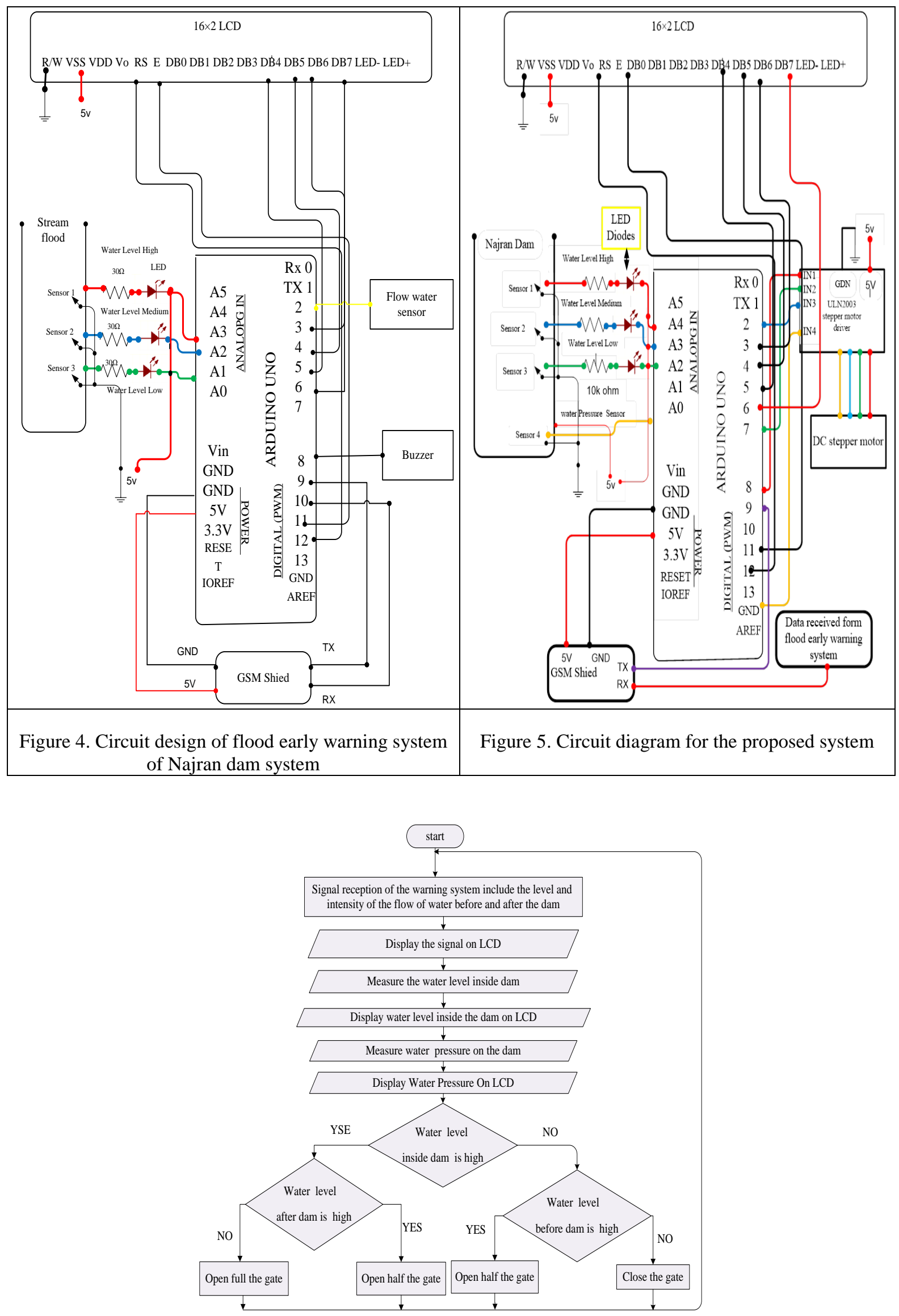

Figure 6. Flow chart of the proposed system 


\begin{tabular}{cl}
\multicolumn{2}{c}{ Table 1. Symbol table } \\
\hline Symbol & \multicolumn{1}{c}{ Description } \\
\hline A & Level inside of dam \\
B & Water stream level before the dam \\
C & Water stream Level after the dam \\
D & The decision \\
X & Close of gate \\
Y & Half-open gate \\
Z & Full - open the gate \\
0 & The lowest level of water \\
1 & The medium level of water \\
2 & The highest level of water \\
\hline
\end{tabular}

Table 2. Tabulator logical control system in the dam gate

\begin{tabular}{|c|c|c|c|c|c|c|c|c|c|c|c|c|c|c|c|c|c|c|c|c|c|c|c|c|c|c|c|c|}
\hline \multirow{3}{*}{ Inputs } & A & 0 & 0 & 0 & 0 & 0 & 0 & 0 & 0 & 0 & 1 & 1 & 1 & 1 & 1 & 1 & 1 & 1 & 1 & 2 & 2 & 2 & 2 & 2 & 2 & 2 & 2 & 2 \\
\hline & B & 0 & 0 & 0 & 1 & 1 & 1 & 2 & 2 & 2 & 0 & 0 & 0 & 1 & 1 & 1 & 2 & 2 & 2 & 0 & 0 & 0 & 1 & 1 & 1 & 2 & 2 & 2 \\
\hline & $\mathrm{C}$ & 0 & 1 & 2 & 0 & 1 & 2 & 0 & 1 & 2 & 0 & 1 & 2 & 0 & 1 & 2 & 0 & 1 & 2 & 0 & 1 & 2 & 0 & 1 & 2 & 0 & 1 & 2 \\
\hline Output & D & $\mathrm{X}$ & $X$ & $X$ & $X$ & $X$ & $X$ & $X$ & $X$ & $X$ & $X$ & $X$ & $X$ & $X$ & $X$ & $X$ & $\mathrm{Y}$ & $\mathrm{Y}$ & $\mathrm{Y}$ & $\mathrm{Z}$ & $\mathrm{Z}$ & $\mathrm{Y}$ & $\mathrm{Z}$ & $\mathrm{Z}$ & $\mathrm{Y}$ & $\mathrm{Z}$ & Z & $Y$ \\
\hline
\end{tabular}

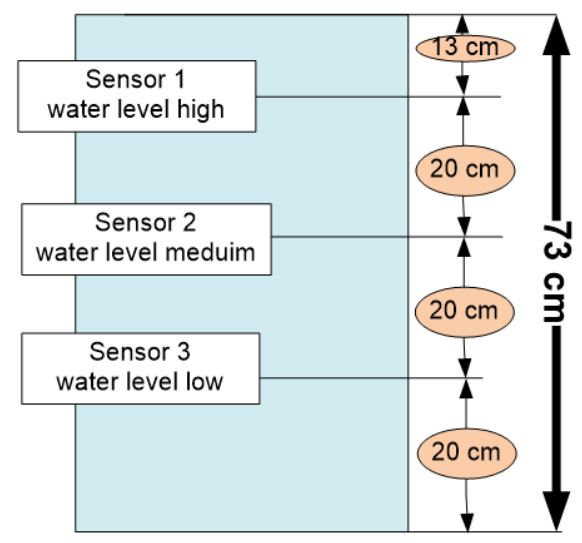

Figure 7. Location of sensor level in Najran dam

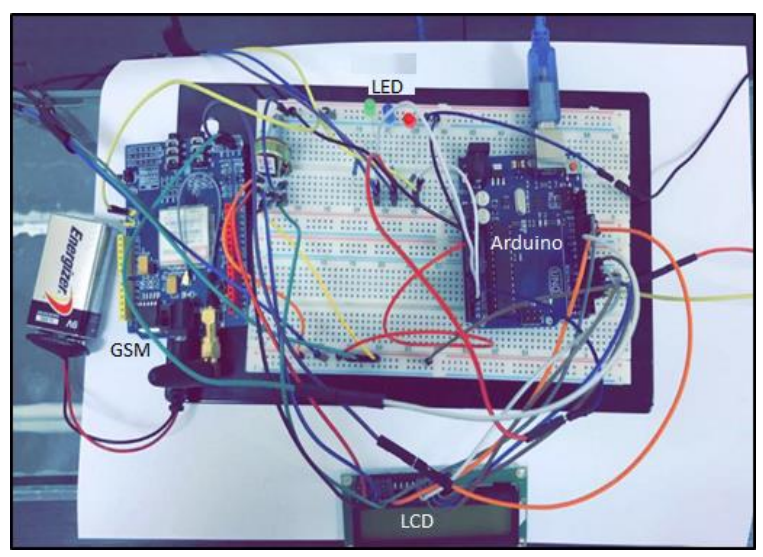

Figure 8. Experimental setup of flood early warning system

\section{RESULTS AND DISCUSSION}

This part of the report is to document and reassure all results obtained from simulations that meet the requirements, objectives of this project. This section discusses the analysis of results, which will be very useful for determining the process of optimization and review to ensure the application of this technique. An experiment was also conducted to verify the flood early warning system, which sends a message containing the water level signal as input to the monitoring system. 
This section discusses the results of the experiment conducted. An experiment was conducted with the effect of high water controlled to validate the model system of the developed system structure as shown Figure 8. Figure 9 shows the monitoring dam prototype. The system architecture and prototype system have been designed advanced. The typical system consists of a medium-sized aquarium tank, water sensor, water pressure sensor, DC stepper motor, Arduino UNO R3 and GSM Shied. The proposed system assembled, tested and verified. The test prototype monitoring system based on flood early warning system were experimented with almost every possible scenario and some very motivating results were found out. To validate the proposed model system, experiment with control. The height of water is measured in the laboratory scale. The water pressure is also measured on the dam barrier by the pressure sensor placed at the dam barrier. In accordance with the Table 1 and Table 2 and through an experiment of the developed system. The procedures for opening and closing the dam gate will be based on conditions as shown in the Table 3.

Table 3. Tabulator logical control system in the dam gate

\begin{tabular}{cl}
\hline Status of dam gate & \multicolumn{1}{c}{ Conditions } \\
\hline Closed & The water level inside the dam is lower than the high \\
Half-open & The level of water in the water stream before the dam is high or the water stream level afar the dam is high \\
Full-open & The level of water in the water stream after the dam was high and the water level inside the dam is high \\
\hline
\end{tabular}

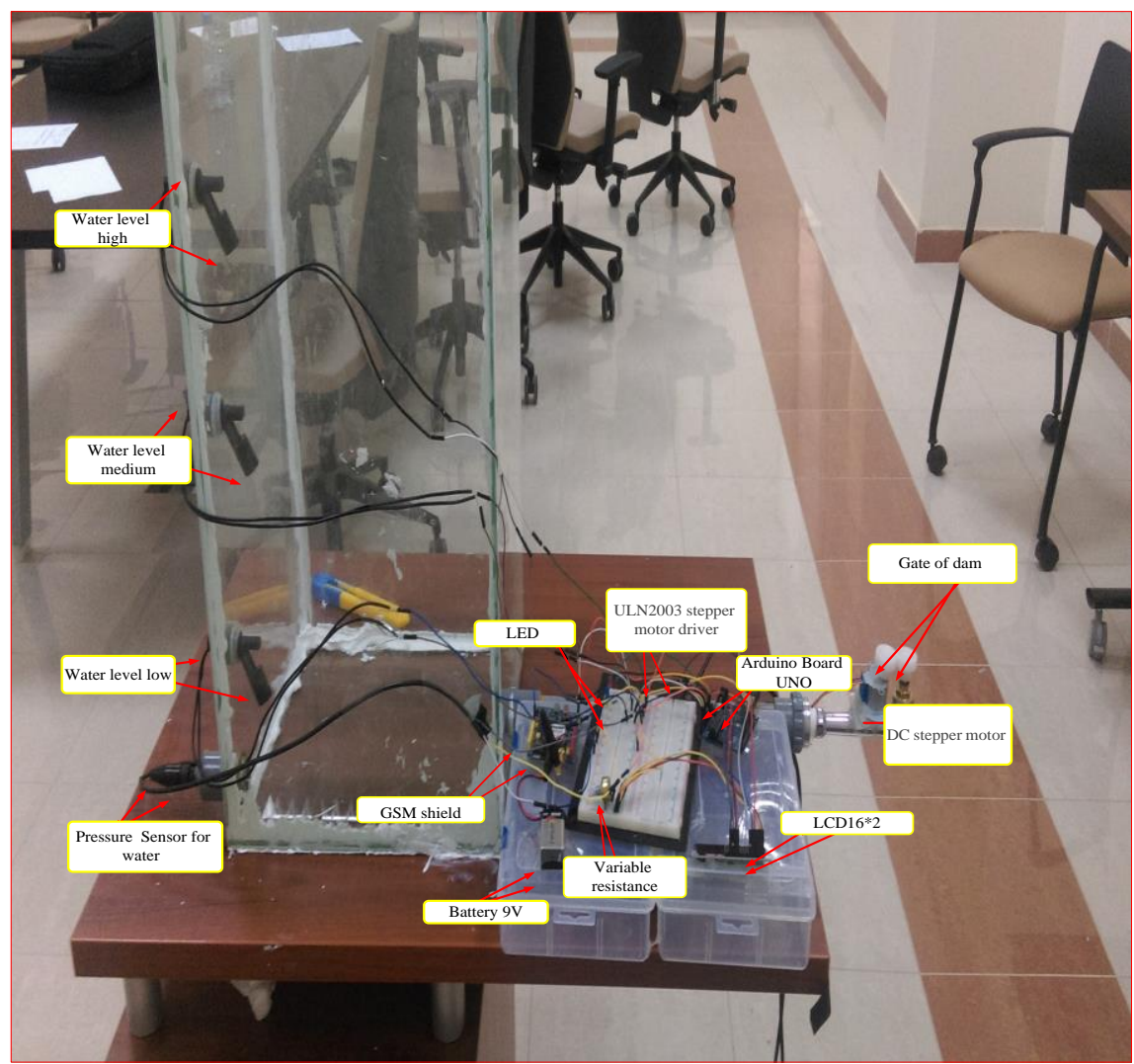

Figure 9. Experimental setup of dam monitoring system

Level sensor number (3) is sensing water level and when water reaches the sensor, it is indicated that the water level in the dam is low. The LED lighting in green and The LCD displays "water level low" and the dam gate is closed. Level sensor number (2) senses water level and when the water reaches a sensor it is indicated that the level is medium. The LED lighting in blue and the LCD display "water level medium" and level DC stepper motor opens the half dam gate. Level sensor (1) senses the water level and when water reaches the sensor gives a signal that the water level is high. The LED lighting in red and the LCD display "water level high" and level DC stepper motor opens the full gate dam. Then the alarm turned ON for the indication that the water level of dam has reached maximum. The gate is also controlled by the flood warning system where a message is received from the flood early warning system that includes the level 
of water in the stream of the flood. When the water level is high, the system closes the dam gate from full open to half open in order to protect the stream from flooding. If the message received is low, the system opens the dam gate. The aim to cooperate between flood early warning system and monitoring dam system to drain the water in the dam and absorb the water coming from the mountains and valleys. In addition, the system measures the water pressure at the dam barrier, which gives information about the water pressure on the dam as well as predict any risks that may occur in the future. Based on the results, the system is already stable in recording the water level accurately, as well as controlling the dam gate, as well as measuring water pressure. The system is reliable because it is able to discharge the dam water with ease and maintain the safety of the dam. The controlling of dam gates was automated by using DC motor which is controlled by Arduino comparing the inputs i.e., the level signal at the inside dam and water stream before and after dam which is obtained from the water level sensors located at the water steam that is far away from the dam. LED and LCD display continuous monitored all information of dam and water level flood. A comparison with recently published works in $[4,8]$ indicated that this system has an advantage of utilizing the Arduino. However, the results presented in [4,6] are using PIC microcontroller and computer. This means that the proposed system is cost-effective and offers simple way of programing and implementation on the real world.

In this work, a prototype for monitoring system of Najran city dam was proposed, implemented and verified with downscaling of 100:1. The main purpose behind this prototyping is to validate the proposed design method of the actual product. Moreover, producing prototypes is often a more instant and low cost process; and as a result, this has allowed designers to provide stakeholders with accurate and useable models before settling upon a particular design. This step must be taken into consideration in deciding how to improve proposed design better within the allocated period and budget.

\section{CONCLUSION}

A successful measure of the water level inside the dam and water level before and after the dam and control of the Najran dam gate has been implemented. All data of dam is displayed on LCD to achieve fourth objective. The results showed that the developed system works with a success rate of $100 \%$. The water sensor has been proven to accurately measure the water level. Also, the stepper motor's ability to control the dam gate, pressure sensor can measure water pressure. It has been concluded that the developed system will assure the effective water drainage system and will help to take immediate action to save lives and minimize damage caused by the flood disaster. With Measure the level of water in the dam and water stream before and after the dam and ensure full control of the dam gate without human intervention, which at the same time help in good discharge of water. Moreover, with a sensitive water pressure sensor on the dam, the water level monitoring process becomes automated, allowing the professional management from flood early warning system to be implemented before, during and after the rain and flood. In conclusion, we confirm a new way to discharge the dam water away from any damage, whether human or material.

\section{REFERENCES}

[1] A. Alhamid, "Najran Valley Dam,” 2004. [Online]. Available: https://en.wikipedia.org/wiki/Najran_Valley_Dam. [Accessed: 01-Jan-2017].

[2] Zakwan H. Zakaria, "Effect of Flooding to People and Enviroment," Prezi, 2015. [Online]. Available: https://prezi.com/fvohig-zflb2/effect-of-flooding-to-people-and-environment/?webgl=0. [Accessed: 10-Jan-2017],

[3] K. Prasanti, C.J. Lakshmi, and C.S. Savalam, "A prototype controlling of barrage gates using arduino under flood conditions," in 3rd International Conference on Electrical, Electronics, Engineering Trends, Communication, Optimization and Sciences (EEECOS), pp. 579-583, Jun. 2016

[4] M. Iyer, "Embedded Dam Gate Control S ystem using 'C' and Visual Basic," Int. J. Comput. Appl., vol. 69, no. 2, pp. 32-37, 2013.

[5] Y.N. Lakshmi, "Automatic Dam Gate Control System with Caution Alarm Using ARM7 Abstract," International Journal of Research, vol. 3, no. 12, pp. 94-102, 2016.

[6] S.M.K. Reza et al., "Microcontroller Based Automated Water Level Sensing and Controlling: Design and Implementation Issue," Proc. of the World Congr. Eng. Comput. Sci. vol. 1, pp. 20-22, 2010.

[7] M. Nicolas and F. Velea, "Automatic Control of a Hydropower Dam Spill Way," Aannals Uuniversity Craiova, Electr. Engineening Series, no. 34, 2010.

[8] S. Muhammad, U. Talha, S. S. Mohani, S. H. Ahmed, and M. Ebrahim, "Design for an Irrigation and Monitoring System of an Automated Dam," in International Multi Confrerence of Engineers and Computer Scientists, vol. 12, pp. 14-17, Mar. 2012.

[9] I. A. Zakarya, F. Naemah, M. Saad, M. Zaim, and M. Nor, "Development of Portable Water Level Sensor for Flood Management System,” ARPN J. Eng. Appl. Sci., vol. 11, no. 8, pp. 5352-5357, 2016. 
[10] S. Azid, B. Sharma, K. Raghuwaiya, A. Chand, S. Prasad, and A. Jacquier, "SMS based flood monitoring and early warning system," ARPN J. Eng. Appl. Sci., vol. 10, no. 15, pp. 6387-6391, 2015.

[11] F. A. Mastor, I. A. Aziz, N. S. Haron, J. Jaafar, and N. N. Ismail, "Pre-flood Warning System Bades on User Mobility," ARPN J. Eng. Appl. Sci., vol. 10, no. 23, pp. 17905-17913, 2015.

[12] M. Banzi and S. Edition, "Getting Started with Arduino," Second Ed. O'Reilly Media, Inc., 2011.

[13] M. Omar et al., "Design and Development of a Smart Parking System," Journal of Automation and Control Engineering, vol. 6, no. 2, pp. 66-69, Dec. 2018.

[14] H. Dahou et al., "Design and Implementation Intelligent Adaptive Front-lighting System of Automobile using Digital Technology on Arduino board," International Journal of Electrical and Computer Engineering (IJECE), vol. 8, no. 1, pp. 521-529, 2018.

[15] T. S. Gunawan, Y. Mahira, S. Munir, M. Kartiwi, and H. Mansor, "Design and Implementation of Portable Outdoor Air Quality Measurement System using Arduino," International Journal of Electrical and Computer Engineering (IJECE), vol. 8, no. 1, pp. 280-290, 2018.

[16] A. H. M. Almawgani, "Design Of Real Time Smart Traffic Light Control System," Int. J. Ind. Electron. Electr. Eng., vol. 6, no. 4, pp. 43-47, 2018.

[17] W. Haszerila, W. Hassan, A. Z. Jidin, S. Asma, C. Aziz, and N. Rahim, "Flood disaster indicator of water level monitoring system," International Journal of Electrical and Computer Engineering (IJECE), vol. 9, no. 3, pp. 1694-1699, 2019.

[18] K. Bhagchandani and D. P. Augustine, "IoT based heart monitoring and alerting system with cloud computing and managing the traffic for an ambulance in India," International Journal of Electrical and Computer Engineering (IJECE), vol. 9, no. 6, pp. 5068-5074, 2019.

[19] T. S. Gunawan, I. Rahmithul, H. Yaldi, M. Kartiwi, and H. Mansor, "Performance Evaluation of Smart Home System using Internet of Things," International Journal of Electrical and Computer Engineering (IJECE), vol. 8, no. 1, pp. 400-411, 2018.

[20] F. Alshehri, A. H. M. Almawgani, A. Alqahtani, and A. Alqahtani, "Smart Parking System for Monitoring Cars and Wrong Parking," 2019 2nd Int. Conf. Comput. Appl. Inf. Secur., pp. 1-6, 2019.

[21] U. P. Glass, "Smart and Efficient Energy Saving System," in IEEE 2019 Smart City Symposium Prague (SCSP), pp. 1-5, 2019.

[22] C.D.L. Cero, K.J.L. Ala, and E.R. Arboleda, "Android Application for Microcontroller-based Reservoir Water Level Monitoring," Indonesian Journal of Electrical Engineering and Informatics (IJEEI), vol. 6, no. 1, pp. 79-85, 2018.

[23] A. R. H. Alhawari, et al., "Design and Development of Electronic Cooling and Heating Pad for Hot and Cold Therapy," ARPN J. Eng. Appl. Sci., vol. 12, no. 24, pp. 7282-7289, 2017.

[24] W. Tariq, M. L. Othman, N. Izzri, A. Wahab, and S. Akhtar, "Smart Monitoring and Controlling of Frequency Deviation by Using MATLAB GUI and ARDUINO DAQ Card," Indonesian Journal of Electrical Engineering and Computer Science (IJEECS), vol. 11, no. 1, pp. 224-232, 2018.

[25] A. Visually and I. Person, "An Ultrasonic System for Assisting Visually Impaired Person," Journal Teknol. (Sciences Eng.), vol. 4, pp. 139-144, 2016.

[26] D. Susanto, K. B. Seminar, and H. Sukoco, "Parallel Processing Implementation on Weather Monitoring System for Agriculture," Indonesian Journal of Electrical Engineering and Computer Science (IJEECS), vol. 6, no. 3, pp. 682-687, 2017. 